\title{
Developments in dental sleep appliances
}

Planmeca is pleased to announce that it is now possible to use the new Planmeca Emerald intraoral scanner to produce Panthera Sleep's Mandibular Advancement Devices (MAD).

Panthera Sleep designs, develops, manufactures and markets high-end mandibular advancement devices (MAD) for the treatment of snoring and/or obstructive sleep apnoea.

The Planmeca Emerald intraoral scanner can now be smoothly integrated into sleep practices' workflows. After scanning the upper and lower arches, first alone and then in occlusion, dentists

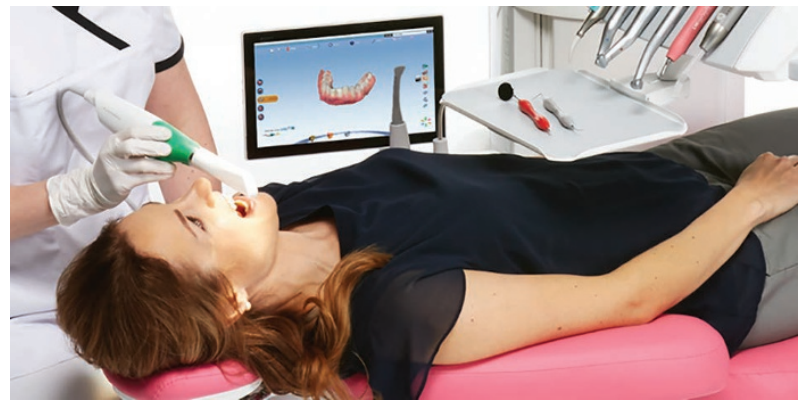

can instantly export the scans to Panthera Sleep.

Headquartered in Quebec City, Canada, Panthera Sleep is a world leader in CAD/CAM dental sleep appliances.

Panthera Sleep has successfully combined creativity, science and know-how to develop its proprietary innovative technology and is now able to offer next-generation products to the sleep industry worldwide. By establishing the Sleep Division, Panthera Dental's goal is clearly to keep helping patients and procure them a most-deserved good night's sleep and quality of life. www.pantherasleep.com www.planmeca.com.

\section{Smell the spring air}

It's springtime! But unfortunately millions of people suffer from seasonal allergies that are triggered by airborne pollen. Runny nose, sneezing and coughing are familiar symptoms and excessive mucus collects in the nose and throat causing unpleasant mouth odour.

A good oral health routine including CB12 mouthwash will help to combat foul smelling gases or volatile sulphur compounds (VSCs) so that patients can maintain fresh breath for longer.

Developed by dentists and clinically proven, CB12 contains low concentrations of zinc acetate and chlorhexidine diacetate and is able to bind chemically to tissues in the oral cavity. This means that $\mathrm{CB} 12$ can provide effective, neutralising action on foul smelling VSCs for up to 12 hours.

CB12 also contains $0.05 \%$ sodium fluoride to encourage good oral health, and is available in refreshing mint menthol and mild mint menthol flavours.

Recommend CB12 and help to reduce oral malodour this spring by contacting $\mathrm{CB} 12$ now.

For more information about CB12 and how it could benefit your patients, visit www.cb12.co.uk.

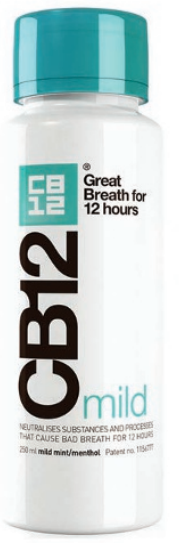

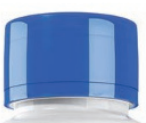

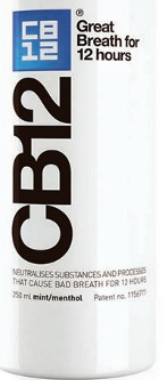

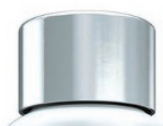

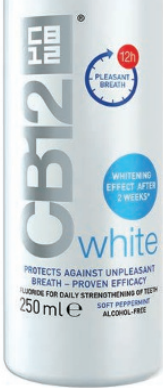

\section{Modern biotech and ancient minerals in a toothpaste}

News on microbiota and immunity is everywhere these days. Shifting oral microbiota to beneficial microorganisms is even possible with toothpastes, containing lactoperoxidase systems. Probiotics are on their way to oral cavity, but you can't keep them alive for a shelf-life time for most oral care products.

But what about mimicking the action of that beneficial flora without using probiotics?

SPLAT, a Russian cosmetic company presented worldwide, have introduced a novel dentifrice formulation containing the lysates of beneficial bifidobacteria that may perform their action through three possible ways simultaneously:

1. Providing signals to mucosal immune cells so that they 'think' that normal microbiota is abundant and slow down their excessive inflammatory activities

2. Bacteriocins from beneficial bacteria lysates act in the same manner as from living microorganisms - they kill abnormal flora, protecting teeth and gums

3. Lysates provide biochemical signal to enhance the survival and growth of the beneficial flora.

There are already two products containing bacterial lysates from SPLAT Oral Care: Sensitive Ultra and Sensitive White - and more being planned. To keep inflammation away, these two new products contain a mineral bischofite - an 'ancient sea salt' which contains a lot of magnesium, providing a shield from periodontitis. Maybe one day this toothpaste will guard you from a sore throat?

For more information, email pavel.nesmiyanov@splat.ru or visit www.splatoralcare.uk. 